May 2019

\title{
First Semester Academic Functioning of College Students: The Role of Stressful and Traumatic Life Events
}

Ashlee Warnecke

University of Louisville, ashlee.warnecke@gmail.com

Richard Lewine

University of Louisville, rrlewi02@louisville.edu

Follow this and additional works at: https://digitalcommons.georgiasouthern.edu/ij-sotl

\section{Recommended Citation}

Warnecke, Ashlee and Lewine, Richard (2019) "First Semester Academic Functioning of College Students: The Role of Stressful and Traumatic Life Events," International Journal for the Scholarship of Teaching and Learning: Vol. 13: No. 2, Article 8.

Available at: https://doi.org/10.20429/ijsotl.2019.130208 


\title{
First Semester Academic Functioning of College Students: The Role of Stressful and Traumatic Life Events
}

\begin{abstract}
The present study sought to better understand the role of stress and trauma history and resiliency among students from low socioeconomic backgrounds and their college academic outcomes. Self-reported history of stressful and traumatic life events, resilience, and demographic factors were collected at orientation for 54 "poor" students as determined by Federal standards. Academic record information was collected at the end of the first semester. The study sample was similar to other students in terms of event exposure, self-reported mental health symptoms, and resilience. Event exposure significantly correlated with course withdrawals, low grades (Fs and Ds), and mean grade point average.. This research has implications for educators, mental health professionals, and college administrators.
\end{abstract}

\section{Keywords}

trauma, resilience, academic outcomes, poverty

\section{Creative Commons License}

c) () $९$

This work is licensed under a Creative Commons Attribution-Noncommercial-No Derivative Works 4.0 License. 


\title{
First Semester Academic Functioning of College Students: The Role of Stressful and Traumatic Life Events
}

\author{
Ashlee Warnecke \& Richard Lewine \\ University of Louisville \\ Received 25 August 2018;Accepted 16 November 2018
}

\begin{abstract}
The present study sought to better understand the role of stress and trauma history and resiliency among students from low socioeconomic backgrounds and their college academic outcomes. Self-reported history of stressful and traumatic life events, resilience, and demographic factors were collected at orientation for 54 "poor" students as determined by Federal standards. Academic record information was collected at the end of the first semester. The study sample was similar to other students in terms of event exposure, self-reported mental health symptoms, and resilience. Event exposure significantly correlated with course withdrawals, low grades (Fs and Ds), and mean grade point average. This research has implications for educators, mental health professionals, and college administrators.
\end{abstract}

\section{INTRODUCTION}

In recent years, the rates and intensity of psychological problems of college students have increased dramatically (Hunt, \& Eisenberg, 20I0). Research reports over half of college students in the United States met criteria for a psychological disorder within the past year (Blanco et al., 2008), with rates of anxiety estimated to be around $40 \%$, and rates of depression around $37 \%$ (Beiter, et al., 20I5). As timing of data collection in past research varies, it is unclear whether students come to campus with diagnosable disorders, or develop them during college. Regardless, mental health symptoms are associated with poor outcomes, including behaviors such as smoking, poor diet, physical inactivity, and poor sleep habits (Doom, \& Haffel, 20I3).

In addition to this, poor mental health is detrimental to the primary focus of most college students, namely academic performance. For example, past research describes those with mental health difficulties as having low levels of educational attainment (e.g., less likely to receive a college degree; Kessler, Foster, Saunders, \& Stang, I995; Mojtabai, et al., 20I5). Although educational attainment is one of the most frequently studied academic outcomes, it is probable that mental health factors also effect academic precursors to drop-out, such as academic performance and time to degree completion.

Students from poverty have traditionally been viewed as especially vulnerable to poor academic outcomes. Early familial poverty has been associated with poor long-term academic outcomes, particularly in those families with the lowest socioeconomic status (SES; Duncan, Brooks-Gunn, Yeung, \& Smith, 1998; Goldrick-Rab, Kelchen, Harris, \& Benson, 2016). This could be related to the historically high rates of reported mental health difficulties in those from low SES backgrounds (World Health Organization, 20I4). In college students, those from a low SES background have also been found to seek help less often for mental health problems (Eisenberg, Golberstein, \& Gollust, 2007). Furthermore, those of low SES backgrounds are likely to report a high number of traumatic events that occurred prior to college, and to report severe traumatic events (Read, Ouimette, White, Colder, \& Farrow, 20 I I).

Based on past research, stress and trauma are important to examine as possible precursors to mental health issues and obstacles to good academic performance. Approximately 66\% of incoming college students report exposure to at least one traumatic stressor (events or situations that involve actual or perceived death, injury, or sexual violence, as well as learning about or witnessing these events; American Psychiatric Association, 20I3). In a study examining enrollment in college students with a history of childhood abuse, dropout rates were higher in students with an abuse history compared to those without an abuse history for all but two semesters. By the end of senior year, only $45 \%$ of abuse survivors were still enrolled, compared to $60 \%$ of non-abuse survivors. Those with a history of multiple abuse types had the highest dropout rates, being enrolled at a rate of only $35 \%$ (Duncan, 2000).

Similarly, in a study that examined the relationship between trauma exposure, post-traumatic stress disorder (PTSD) and drop-out rates, those with trauma exposure and PTSD symptoms dropped out at a rate of $35 \%$, while those with trauma exposure and no PTSD symptoms dropped out at a rate of $20 \%$ (Boyraz, Granda, Baker, Tidwell, \& Waits, 20I5; Boyraz, Horne, Owens, \& Armstrong, 20I3). Syndromal distress after trauma, though, does not seem to be necessary to increase dropout rates, as dropout rates increase in the absence of self-reported distress or mental health disorders, including PTSD (Duncan, 2000; Hardaway, Larkby, \& Cornelius, 2014).

Although many students with a history of adversity from low SES backgrounds show poor academic functioning, not all students have this experience. Furthermore, although Martin and Elmer (1992) reported a history of severe abuse led to poor groupwise outcomes across domains, they also found a range of individual differences, including some individuals who completed higher education and obtained jobs, while having families and strong social ties. As not all students with a history of stressors have poor outcomes, the question of what the difference is between those students who have poor outcomes and those who do not arises.

One possible answer to this question is resilience. Resilience has been defined as good outcomes, despite threats to development or adaptability (Masten, 200I). While resilience was once thought to be rare, much research now indicates it is common following adversity (Bonanno, \& Mancini, 2008). Recent research, though, has again called into question how common resilience is following highly stressful life events, suggesting that in some cases it may be the least common outcome (Infurna, \& Luthar, 2016). 
This underscores the importance of continuing to examine resiliency processes to gain a better understanding of resilience.

Long-term studies of resilience point to its malleability over time. Emerging adulthood, the developmental period of many college students, is a unique time, with possibilities for changing the life course. Indeed, opportunities themselves may create the necessary conditions for positive change (Masten, Obradović, \& Burt, 2006). Core childhood resources, as well as unique resources of emerging adulthood, are related to the successful transition to adulthood, including academic attainment (Masten et al., 2004). Perhaps due to malleability of resilience over time, approximately half of those with a history of traumatic and non-traumatic stressors show poor academic outcomes, despite the potential resilience needed to gain admission to college.

This study examines both potential obstacles (stress and trauma) to and potential facilitators (resilience) of academic success in a group that has historically been considered particularly vulnerable - students coming from families below the $150 \%$ federal poverty level. Focus in this study is unique as it is on within group heterogeneity of a significantly disadvantaged group, as compared to the typical strategy of comparing disadvantaged to advantaged students. Aims include providing descriptive information on this unique sample, as well as examining relationships between a history of stressful and traumatic events, academic outcomes, and resilience.

\section{METHODS}

\section{Population and Sample Selection}

A sample of 57 students from the Fall 2016 incoming class at a southern public research university in the United States were recruited. Of the 57, three were under the age of 18 and not eligible per IRB approved protocol, resulting in a final sample of 54 students. The program from which the sample was recruited provides complete financial assistance (tuition, room, board, and books) to students who apply for the program and are at the $150 \%$ federal poverty level. In addition to the standard application, students applying for the special program must submit an additional essay, meet minimal academic requirements ((20 ACT composite score (a standardized test used for college admissions) and 2.5 High School grade point average)), complete a Free Application for Federal Student Aid (FAFSA), have a complete financial aid file, and meet certain grant requirements.

\section{MEASURES}

\section{Demographic Form}

The demographics form included basic personal information such as sex/gender, birthdate, and age. Additional collected data included the student's academic, family and social information.

\section{Life Stressor Checklist-Revised (LSC-R).}

The LSC-R was designed to screen for traumatic events, as well as events that may be considered stressful but not traumatic (Wolfe, et al., 1996). For each of the 30 events an individual endorses, follow-up questions assess for event-related distress. We used all three accepted scoring methods for this study: frequency of traumatic events only; assigning one point for each endorsed event (scores ranging from 0-30); and total score of assigned weights for endorsed events (scores ranging from 0 - I50, reflecting an individual's subjective rating of how the event affected life in the past year). Test-retest reliability ranges across items. For example, a Kappa of .52 has been reported for physical abuse, while a Kappa of .97 has been reported for miscarriage (McHugo et al., 2005). Concurrent validity has been supported with other measures of stress and trauma, such as the Impact of Event Scale-Revised and the Symptom Checklist 90-Revised (Ungerer et al., 20l0). In the present sample, the internal consistency of the measure was fair at .76 .

\section{Beck Anxiety Inventory (BAI).}

The BAI (Beck, 1990) is a 21 -item self-report measure designed to assess anxiety symptoms. Each of the items is measured on a 4 -point Likert scale $(0=$ none to $3=$ frequently). Total scores range from 0 to 63 . The internal consistency of the BAl ranges from .81 to .95 . Internal consistency in the present sample was similar, at 96.

\section{Beck Depression Inventory-II (BDI-II)}

The BDI-II is a $2 \mathrm{I}$-item self-report instrument designed to measure depressive symptoms. The items are rated on a 4-point Likert scale $(0=$ none to $3=$ frequently). Total scores range from 0 to 63 . The internal consistency of the BDI ranges from 81 to .95. Internal consistency in the current sample was similar, at .91.

\section{Primary Care PTSD Screen (PC-PTSD)}

The PC-PTSD is a brief (4-item) commonly used screener for PTSD (Prins et al., 2003). Individuals respond "yes" or "no" to each of the items and can receive a score from 0-4. The optimal cutoff score for potential clinical diagnosis of PTSD is 3. Past research found that the PC-PTSD outperformed a well-established measure of PTSD, the PTSD-Checklist (PCL) in terms of overall quality, sensitivity (.78 compared to .46) and specificity (.87 compared to .79; Prins et al., 2003). Internal consistency for the present sample could not be conducted for this measure due to missing data.

\section{Brief Resilience Scale}

The Brief Resilience Scale measures an individual's self-reported ability to bounce back from stressful events (Smith et al., 2008). Participants rate on a scale of I (strongly disagree) to 5 (strongly agree) each of 6 statements. In student samples, average scores have been found to be between 3.53 and 3.57. Test-retest reliability has been reported to be .69 (Smith et al., 2008). Internal consistency in the present sample was fair at .75. Expected Academic Difficulty, Perceived Academic
Preparedness, Academic Perseverance subscales
from the Beginning College Survey of Student En-
gagement (BCSSE).
The BCSSE is administered to incoming students prior to the start of fall classes. It has 42 items and 9 subscales, 3 of which were used in the current study: Expected Academic Difficulty (4 items), Perceived Academic Preparedness (7 items), and Academic Perseverance (6 items) (BCSSE, 20 I0). Response options range from I (lowest) to 6 (highest). In the present sample, internal consistency for the scales were:Academic Perseverance .73; Perceived Academic Difficulty .57; Perceived Academic Perseverance 78.

\section{Grade Point Average (GPA)}

GPA is a calculated average of letter grades earned in college following a 0 to 4.0 scale. GPA was collected at the end of the first semester of the first academic year from the student's official transcript. 


\section{Number of $D$ and $F$ grades earned; Number of course withdrawals}

The number of failing grades ( $D$ and $F$ grades) earned and number of course withdrawals for each student was counted at the end of the first semester (collected from official transcript).

\section{DATA COLLECTION}

Baseline questionnaires (i.e., demographic form, LSC-R, BAI, BDIII, PC-PTSD, and Brief Resilience Scale) were administered as part of the program at an orientation session at the beginning of the academic year. Students received information about the purpose of data collection, potential for risks and benefits of participation, confidentiality, procedures for collection of completed packets, and guidelines for discontinuing participation. Packets including an informed consent document and baseline self-report measures were distributed. Students were given as long as they needed to complete the packets and returned them when finished.These packets (which also included questionnaires on cognitive coping style not discussed in this study), along with pre-admission essays (not discussed in this study), represent the entirety of one type of data collected - program forms and information. The other type of data collected were non-program documents, including transcripts and BCSSE data. These data were collected following the end of the first semester. Students were sent a letter at that time reminding them of the collection of these data and indicating they could contact researchers to withdraw consent. No students withdrew consent. IRB approval was obtained to use both classes of data.

\section{Data Analysis Plan}

\section{Analysis Decisions}

Data preparation and analyses were conducted using SPSS v22.0 (SPSS IBM, Armonk, NY, 20I3). Data were examined visually through plots and tables to determine if there was any identifiable pattern to missing data. When examining responses on the LSC-R, missing data were identified for variables asking about the individual's experiences with their own children. Given the large number of students who did not respond to these questions, total LSC-R scores were still calculated for these students, with missing data being assumed to be zero (event not experienced). When calculating the weighted score for the LSC-R, six students who indicated they experienced an event did not answer the required follow-up question for at least one endorsed event. These students were removed from analyses for this variable. When considering the PTSD screener, only 29 students had a total score for the measure. Due to the small sample size, only descriptive information will be provided. No other systematic bias was identified. To inform understanding of these measures in this unique sample, all scales were examined for signal items that may have influenced results. No signal items were identified.

As many variables included in the study did not have normal distributions, median and interquartile range are provided as a measure of central tendency when appropriate. For other variables, percent of sample is provided. Given the limited range of fall course withdrawals (0-2), this was collapsed into a dichotomous variable - did/did not withdraw from a course. Similarly, for ethnicity, categories were collapsed to create a dichotomous variable (white, non-white). Given the need to use non-parametric tests, Spearman's rank correlations are provided for all variables with the exception of gender, ethnicity and fall course withdrawals. Point-biserial correlations are provided for these variables. No multicollinearity was identified, based on examination of variance inflation factor. Regressions are used to assess for the presence of indirect effects (Baron, \& Kenny, 1986). Examination of beta weights and change in predictive value of the predictor variables is used to determine the possible presence of indirect effects.

\section{Sample Size and Statistical Power}

A post-hoc power analysis using G-Power software was conducted. With an alpha $=.05$, sample size $=54$, and medium effect size $=.15$ (Cohen, 1988), the achieved power was .79. This is sufficient for the analyses discussed in this manuscript, including regression analyses.

\section{RESULTS \\ Demographic Characteristics}

See Table I for detailed descriptive information. The majority of the sample was 18 years old, female, and White. Self-reported median high school GPA was 3.66 and self-reported median ACT score was 25.89. Most students reported their primary source of income to be themselves (64.8\%), and students were primarily employed on campus $(79.6 \%)$ for II-20 hours/week $(50 \%)$ while completing a median of 14 credit hours and living with other students $(85.2 \%)$. The majority $(53.7 \%)$ of parents were employed, with annual reported income of parents typically being less than \$9, 999 (37\%). The majority (85.2\%) of participants were first-generation four-year college students with $40.7 \%$ of parents earning a high school diploma.

\section{Measure Descriptive Information}

Table 2 summarizes descriptive information for all measures. Note median scores are reported for data from the present study, though mean scores from other research are discussed at times to provide context for the similarities and differences between this sample and other college samples. For the unweighted LSC-R, the median number of stressful and traumatic life events was 3.00 .The majority of the sample (93\%) endorsed at least one stressful and traumatic life event. Fifty-seven percent reported experiencing at least one traumatic event (median $=2$ ). This is lower than expected and inconsistent with other studies of college students, though these studies used different measures, or the same measure as this study with different scoring (Anders, Frazier, \& Shallcross, 2012; Elhai et al., 2012; Freeman, \& Fowler, 2009; Read et al., 201 I). With respect to frequency, females reported more total events than males and students identifying as multiracial endorsed more events than other ethnicities. With respect to degree of distress, males scored more highly than females, and those students identifying as African American/Black scored higher than students from other ethnic groups.

The most commonly endorsed life event was parental separation/divorce (68.5\%). Other commonly endorsed events included death of a close other (not unexpected) (48.1\%), having a close family member sent to jail (35.2\%), and witnessing familial violence before age 16 (31.5\%). See Table 3 for a full breakdown of endorsed events.

Overall, the sample reported minimal symptoms of mental health difficulties. However, two of the measures (BAI and PCPTSD) had ranges that covered the spectrum of healthy to un- 


\begin{tabular}{|c|c|}
\hline Variable & $\begin{array}{l}\text { Median (IQR) or } \\
\% \text { of Sample (n) }\end{array}$ \\
\hline Age 18 & $96.30 \%(52)$ \\
\hline Female Gender & $61.10 \%(33)$ \\
\hline High School GPA & $3.8(.57)$ \\
\hline ACT & $25(6)$ \\
\hline Completed Enrolled Hours & $14(4.50)$ \\
\hline \multicolumn{2}{|l|}{ Ethnicity } \\
\hline White/European & $57.40 \%(3 \mathrm{I})$ \\
\hline African American/Black & $13.00 \%(7)$ \\
\hline Hispanic/Latino/a & $5.06 \%(3)$ \\
\hline Asian/Pacific Islander & $9.03 \%(5)$ \\
\hline Multiracial & $11.10 \%(6)$ \\
\hline \multicolumn{2}{|l|}{ Income Source } \\
\hline Parent/Guardians & $22.20 \%(12)$ \\
\hline Self (Scholarships/Grants/Work) & $64.80 \%(35)$ \\
\hline \multicolumn{2}{|l|}{ Employed } \\
\hline On-Campus & $79.60 \%(43)$ \\
\hline Off-Campus & $18.50 \%(10)$ \\
\hline \multicolumn{2}{|l|}{ On-Campus Employment Hours } \\
\hline I-I0 hours/week & $27.80 \%(15)$ \\
\hline II-20 hours/week & $50.00 \%(27)$ \\
\hline Greater than 30 hours/week & $1.90 \%(1)$ \\
\hline \multicolumn{2}{|l|}{ Housing } \\
\hline Alone & $3.70 \%(2)$ \\
\hline With other students & $85.20 \%(46)$ \\
\hline With parent/relative/guardian & $11.10 \%(6)$ \\
\hline \multicolumn{2}{|l|}{ Parental Household Income } \\
\hline Less than $\$ 9,999$ & $37.00 \%(20)$ \\
\hline$\$ 10,000-\$ 19,999$ & $27.80 \%(15)$ \\
\hline$\$ 20,000-\$ 39,999$ & $25.90 \%(14)$ \\
\hline$\$ 40,000-\$ 59,999$ & $1.90 \%(1)$ \\
\hline \multicolumn{2}{|l|}{ Primary Source of Income for Parents } \\
\hline Disability & $24.10 \%(13)$ \\
\hline Employment & $53.70 \%(29)$ \\
\hline Inheritance & $1.90 \%(1)$ \\
\hline Public Assistance & $1.90 \%(1)$ \\
\hline Other & $7.40 \%(4)$ \\
\hline \multicolumn{2}{|l|}{ Highest Education Level Parents } \\
\hline Did not complete high school & $13.00 \%(7)$ \\
\hline High school diploma & $40.70 \%(22)$ \\
\hline Attended college, no degree & $18.50 \%(10)$ \\
\hline Associate's degree & $13.00 \%(7)$ \\
\hline Bachelor's degree & $11.10 \%(6)$ \\
\hline Advanced degree & $3.80 \%(2)$ \\
\hline
\end{tabular}

healthy. Of the 29 students who completed the PC-PTSD, 6 had scores meeting the cutoff score for potential PTSD, a percentage similar to those found in validity studies of the measure (Prins et al., 2003; Prins et al., 20I5). Scores on the BAI and BDI were lower than scores found in some college samples, with other research reporting mean scores of 9.62 on the BAI (Jansen, Motley, \& Hovey, 2010) and mean scores on the BDI as I2.75 and II.86 (Carmody, 2005; Steer, \& Clark, 1997, respectively).

\begin{tabular}{|c|c|c|}
\hline Variable & $\begin{array}{l}\text { Median (IQR) or } \\
\% \text { of Sample (n) }\end{array}$ & $\begin{array}{c}\text { Range } \\
\text { (when applicable) }\end{array}$ \\
\hline LSC-R Unweighted & $3.00(5)$ & $0-12$ \\
\hline \multicolumn{3}{|l|}{ Gender } \\
\hline Female & $4.50(5.75)$ & $0-12$ \\
\hline Male & $3.00(3.00)$ & $0-9$ \\
\hline \multicolumn{3}{|l|}{ Ethnicity } \\
\hline White/European & $4.00(5.00)$ & $\mid-1 I$ \\
\hline African American/Black & $2.00(5.00)$ & $0-8$ \\
\hline Hispanic/Latino/a & $3.00(5.00)$ & $1-12$ \\
\hline Asian/Pacific Islander & $2.00(5.00)$ & $0-8$ \\
\hline Multiracial & $5.00(4.25)$ & $1-22$ \\
\hline LSC-R Weighted & $7.50(12.75)$ & $0-36$ \\
\hline \multicolumn{3}{|l|}{ Gender } \\
\hline Female & $6.00(16.00)$ & $0-36$ \\
\hline Male & $10.00(16.00)$ & $0-36$ \\
\hline \multicolumn{3}{|l|}{ Ethnicity } \\
\hline White/European & $8.50(15.75)$ & $0-36$ \\
\hline African American/Black & $11.00(10.00)$ & $\mathrm{I}-23$ \\
\hline Hispanic/Latino/a & $7.00(7.00)$ & $3-13$ \\
\hline Asian/Pacific Islander & $4.00(30.00)$ & $\mathrm{I}-36$ \\
\hline Multiracial & $3.50(16.50)$ & $\mathrm{I}-22$ \\
\hline Traumatic Events & $2.00(3)$ & $1-11$ \\
\hline BRS & $3.50(1)$ & $2.17-5.00$ \\
\hline \multicolumn{3}{|l|}{ BCSSE } \\
\hline Expected Academic Difficulty & $30.00(\mathrm{II})$ & $12-48$ \\
\hline Academic Preparedness & $47.14(9.75)$ & $24-60$ \\
\hline Academic Perseverance & $48.00(I 0.7 I)$ & $28-60$ \\
\hline GPA & $3.00(1.40)$ & $0.00-4.00$ \\
\hline $\mathrm{D}$ and $\mathrm{F}$ Grades & $0(I)$ & $0-4$ \\
\hline 0 & $63.00 \%(n=34)$ & \\
\hline $\mathrm{I}$ & $18.50 \%(n=10)$ & \\
\hline 2 & $11.10 \%(n=6)$ & \\
\hline 3 & $3.70 \%(n=2)$ & \\
\hline 4 & $1.90 \%(n=1)$ & \\
\hline Withdrawals & $0(1)$ & $0-2$ \\
\hline 0 & $74.10 \%(n=40)$ & \\
\hline 1 & $22.20 \%(n=12)$ & \\
\hline 2 & $1.90 \%(n=1)$ & \\
\hline BAI & $8.50(28)$ & $0-60$ \\
\hline BDI & $6.00(11)$ & $0-4 \mid$ \\
\hline PTSD Screener & $I(2)$ & $0-4$ \\
\hline \multicolumn{3}{|c|}{$\begin{array}{l}\text { Note. } N=54 \text { except for D and F grades (53), withdrawals, gender, ethnicity, } \\
\text { BAI (52), BDI (5I), LSC-R Weighted ( } 48) \text {, traumatic events ( } 3 \text { I students } \\
\text { who endorsed at least one event), PTSD Screener ( } 29 \text { students who en- } \\
\text { dorsed question one). }\end{array}$} \\
\hline
\end{tabular}

The median score on the BRS was similar to the mean score found in other samples of college students (Smith, et al., 2008). In contrast to median scores for Perceived Academic Difficulty, median scores for Perceived Academic Preparedness and Academic Perseverance were slightly higher than mean scores of the overall student sample at the university from which the sample came, as well as first-generation mean scores. However, this difference was only three to four points in all cases. Expected Aca- 


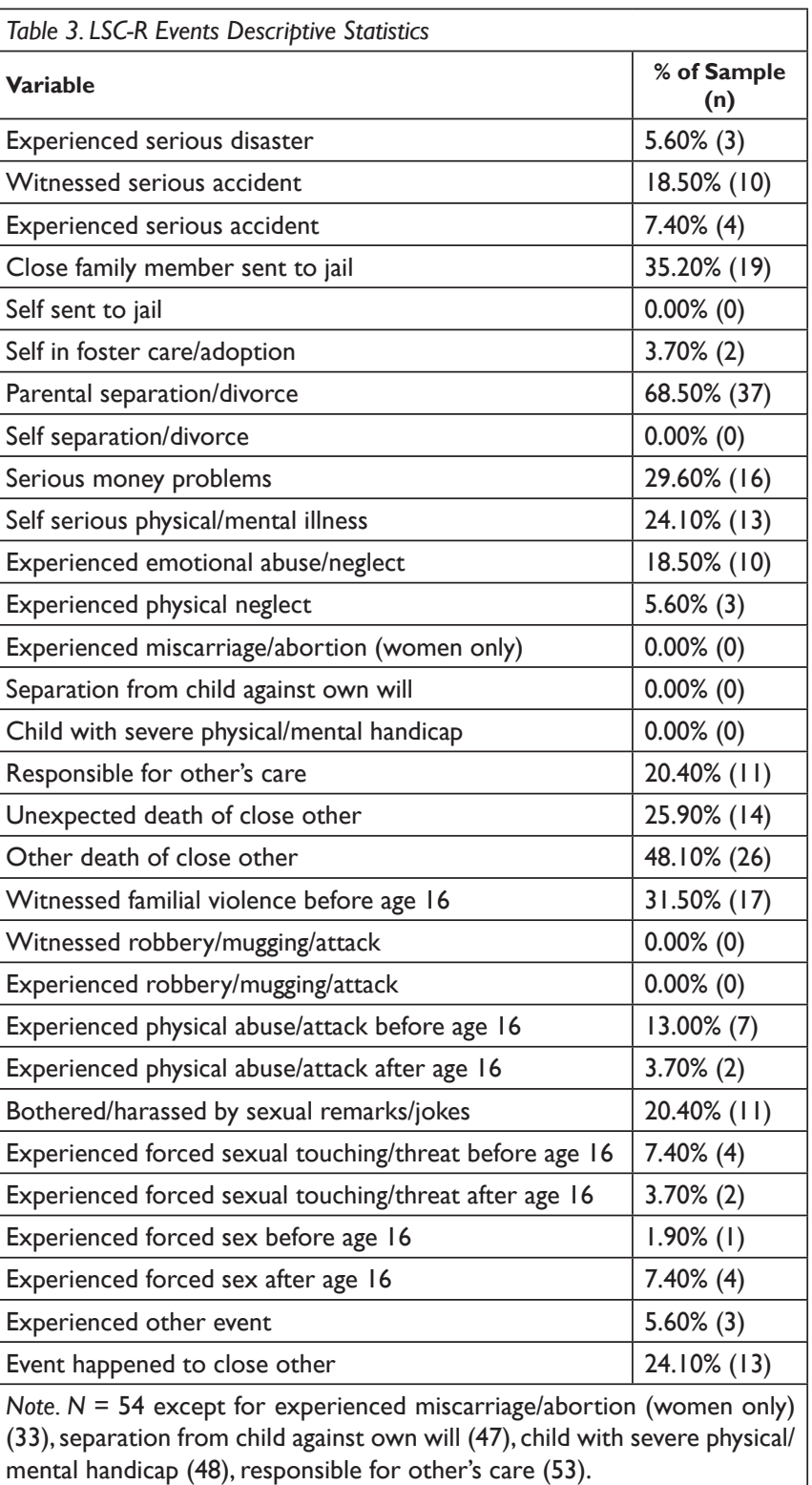

demic Difficulty scores were similar to the overall sample from the university and nearly identical to those in the first-generation college student subset (University of Louisville, 2016). Comparison scores for the BCSSE were obtained from a public report released annually by the university, which summarizes aggregate BCSSE data (University of Louisville, 2016).

The median fall semester GPA was 3.00. This is similar to first-year GPA reported in other research (Bachrach, \& Read, 2012; Harackiewicz, Barron, Tauer, \& Elliot, 2002). Most students did not withdraw from a course (range $0-2$ ) and received no D/F grades.

\section{Correlations}

See Table 4 for full correlation matrix. Of note, anxiety and depression were significantly positively correlated $(\varrho=.65, p<$ $.0 \mathrm{I})$, and resilience was significantly negatively correlated with both anxiety and depression $(\varrho=-.48, p<.01$ and $\varrho=-.33, p<$ $.0 \mathrm{I}$, respectively). Anxiety and depression were both significantly positively correlated with the unweighted LSC-R score $(\varrho=.40$, $p<.0 \mathrm{I}$ and $\varrho=.30, p<.05$, respectively).
Self-reported resilience was significantly positively correlated with both Academic Perseverance and Academic Preparedness $(\varrho=.43, p<.0 \mathrm{I}$ and $\varrho=.27, p<.05$, respectively). Contrary to expectations, no resiliency variables were significantly correlated with the predictor or outcome variables. Notably, the unweighted LSC-R score although significantly correlated with fall semester course withdrawals ( $r p b=.30, p<.05)$, but was not associated with other outcome variables. All outcome variables (Fall GPA, Fall D/F Grades, and Fall Course Withdrawals) significantly correlated with one another.

In summary, the analyses revealed expected correlations, such as those between anxiety and depression, mental health and self-reported resilience, mental health and self-reported life experiences, and the academic outcome variables. Correlations that reached the level of statistical significance fell in the medium to large effect size range. It is noteworthy that many correlations that did not reach statistical significance also fell in the medium effect size range, including correlations between the weighted LSC-R score and both Fall GPA and Fall D/F grades, as well as depression and Fall Course withdrawals and Expected Academic Difficulty and Fall GPA.

\section{Regressions}

In most cases, correlations were not significant and, therefore, regression analyses were not completed, with one exception, LSC-R unweighted and course withdrawals. As Fall course withdrawals had to be dichotomized, a logistic regression was used. Due to the exploratory nature of this study, two other regression analyses were completed. Both of these included the LSC-R weighted score as the predictor, with one regression for the outcome variable of D/F grades $(\varrho=-.25, p=.08$ with LSC-R weighted) and the other for the outcome variable of Fall semester GPA ( $\varrho=.26, p=.07$ with LSC-R weighted). In both regressions, the only control variable included was high school GPA $(\varrho=.32, p=.03$ with LSC-R weighted $)$ and the only potential indirect effect included was Perceived Academic Preparedness $(\varrho=$ $.24, p=.09$ with LSC-R weighted). These were included based on correlations approaching significance. Given that assumptions are violated, these results will be interpreted with caution.

The first regression, using logistic regression to predict Fall course withdrawals from total number of stressful and traumatic life events reported (unweighted) was significant, Wald's $X^{2}(I)=$ $4.72, p<.05, \beta=.13, \mathrm{OR}=1.24,95 \%$ Confidence Interval (I.0I, I.5I). For each additional stressful and traumatic event the student was exposed to, they were $24 \%$ more likely to withdraw from a course in the Fall semester.

The second regression, predicting Fall D/F grades from total number of stressful and traumatic life events reported (weighted) while controlling for high school GPA and perceived academic preparedness (potential indirect effect) was significant, $F(3,45)$ $=1.87, p<.05$. The final model predicted $14 \%$ of the variance in fall D/F grades, with $11 \%$ of that being predicted by self-reported stressful and traumatic life events. Based on comparison to a regression run without the potential indirect variable in the model, adding in this variable did not alter the predictive value of stressful and traumatic life events (no change in standardized beta or $r$-square change for predictor variable). See Table 5 for full summary of regression two - predicting Fall D/F grades.

The final regression, predicting Fall GPA from total number of stressful and traumatic life events reported (weighted) while 
Table 4

Correlation Matrix

\begin{tabular}{|c|c|c|c|c|c|c|c|c|c|c|c|c|c|c|c|c|}
\hline V ariable & 1 & 2 & 3 & 4 & 5 & 6 & 7 & 8 & 9 & 10 & 11 & 12 & 13 & 14 & 15 & 16 \\
\hline 1. High School GPA & - & .16 & .04 & .11 & .06 & .03 & .07 & -.07 & -.07 & .07 & -.02 & .06 & $-.32 *$ & .22 & $-.29 *$ & -.04 \\
\hline 2. $\mathrm{ACT}$ & - & - & .27 & -.03 & $-.37 *$ & .06 & -.14 & .08 & .04 & -.10 & .18 & $.36^{* *}$ & -.17 & .08 & .05 & .01 \\
\hline 3. Enrolled Hours & - & - & - & -.14 & .05 & -.12 & -.10 & -.05 & .21 & .11 & .03 & .00 & -.14 & .01 & .03 & .17 \\
\hline 4. Sex & - & - & - & - & -.12 & $.47 * *$ & .21 & $-.34 * *$ & .16 & .03 & -.20 & $.36^{*}$ & .02 & .21 & -.24 & .05 \\
\hline 5. Ethricity & - & - & - & - & - & -.02 & .07 & -.19 & .07 & -.01 & -.10 & -.25 & -.22 & .05 & -.07 & -.11 \\
\hline 6. Anxiety & - & - & - & - & - & - & $.65^{* *}$ & $-.48 * *$ & .02 & -.17 & -.08 & $.40 * *$ & -.08 & -.03 & -.09 & .04 \\
\hline 7. Depression & - & - & - & - & - & - & - & $-.33^{*}$ & .06 & .02 & -.04 & $.30 *$ & -.05 & -.10 & .01 & .23 \\
\hline 8. Resilience & - & - & - & - & - & - & - & - & .01 & $.43 * *$ & $.27 *$ & -.01 & .18 & .09 & -.01 & -.11 \\
\hline 9. Academic Difficulty & - & - & - & - & - & - & - & - & - & .05 & -.04 & .00 & .12 & .21 & -.08 & .06 \\
\hline 10. Academic Perseverance & - & - & - & - & - & - & - & - & - & - & $.39 * *$ & .08 & .13 & .15 & -.04 & .06 \\
\hline 11. Academic Preparedness & - & - & - & - & - & - & - & - & - & - & - & .09 & .24 & .00 & -.01 & -.01 \\
\hline 12. LSC-R Unweighted & - & - & - & - & - & - & - & - & - & - & - & - & -.08 & -.01 & .07 & $.30 *$ \\
\hline 13. LSC-R Weighted & - & - & - & - & - & - & - & - & - & - & - & - & - & .26 & -.25 & -.15 \\
\hline 14. Fall GPA & - & - & - & - & - & - & - & - & - & - & - & - & - & - & $.80^{* *}$ & $-.48 *$ \\
\hline 15. Fall D/F Grades & - & - & - & - & - & - & - & - & - & - & - & - & - & - & - & $.51^{* *}$ \\
\hline 16. Fall Course Withdrawals & - & - & - & - & - & - & - & - & - & - & - & - & - & - & - & - \\
\hline
\end{tabular}

Note. $* p<.05 * * p<.01$

controlling for high school GPA and perceived academic preparedness (potential indirect effect) was significant, $F(3,45)=$ $2.21, p<.01$. The final model predicted $16 \%$ of the variance in Fall GPA, with $14 \%$ of that being predicted by self-reported stressful and traumatic life events. Based on comparison to a regression run without the potential indirect variable in the model, adding in this variable did not alter the predictive value of stressful and traumatic life events (no change in standardized beta or r-square change for predictor variable). See Table 6 for full summary of regression two - predicting Fall GPA.

In summary, a logistic regression predicting Fall course withdrawals from total number of stressful and traumatic life events reported (unweighted) was significant, with each additional stressful and traumatic event leading to a $24 \%$ increase in the likelihood of withdrawing from a course in the Fall semester. $A$ regression predicting fall $D / F$ grades from total number of stressful and traumatic life events reported (weighted) was significant, with $11 \%$ of the variance in Fall D/F grades being predicted by self-reported stressful and traumatic life events. The final regression, predicting Fall GPA was significant with $14 \%$ of the variance in Fall GPA being predicted by self-reported stressful and traumatic life events.

\section{DISCUSSION}

The purpose of this study was to better understand the relationship between a history of stressful and traumatic life events and academic outcomes in a sample of low SES college students, and the potential role of resilience in this relationship. We found that the students in this study reported less stressful and traumatic life events than expected. Also contrary to hypotheses, there were no significant correlations between resilience and either a history of stressful and traumatic life events or academic outcomes. Regression analyses indicate that adding resilience (self-reported expected academic perseverance) to the model did not change the relationship between stressful and traumatic life events and an academic outcome.

One noteworthy aspect of this study was the novelty of the sample - a group of college students selected on the basis of their poor economic backgrounds. Despite this, the students were largely similar to samples of college students participat- ing in other research. For example, in the case of life events, the present study found that $57 \%$ of the sample experienced a traumatic event, while past research reports this is typically around 65\% (Elhai et al., 2012; Freeman, \& Fowler, 2009; Read et al., 20I I).A similar pattern was noted for total number of stressful and traumatic life events, as well as depression/anxiety symptoms. One possible explanation for our findings is the timing of data collection. Baseline data collection occurred before the first semester. Most other research has collected data on students further into their academic careers (Boyraz et al., 2013; Boyraz et al., 2015). Past work describes the "freshman myth," wherein the expectations of freshmen who are entering college are, essentially, too positive and optimistic, when compared to the actual experience of being in college (Ailes, Alvarado, Amundson, Bruchey, \& Wheeler, 2017; Baker, McNeil, \& Siryk, 1985; Krieg, 2013; Watkins, 1978). It is possible the results in the present study were subject to overly optimistic expectations, leading to lower scores on self-reported mental health than anticipated. In terms of stressful and traumatic life events, collecting data later in the college career provides more time for students to experience events.

Although this explanation is possible, when considering variables for which a direct comparison could be made to college students who were not from a low socioeconomic background, namely variables from the BCSSE, the current sample was nearly identical to the rest of the students at the university. Therefore, it is also possible the current research supports a different hypothesis: students from low socioeconomic backgrounds do not differ from other students when considering event history, mental health symptoms, or resiliency at the beginning of college. This is contrary to research describing a relationship between low socioeconomic and traumatic event exposure (Read et al., 20l I). Based on this, one would expect the students in this study would report a higher number of events, particularly traumatic events. Future work in this area would benefit from direct comparisons within the same sample at the same time period to better understand whether students from low SES backgrounds differ from other students.

Similarly, we did not find the expected correlations between stressful and traumatic life event history, resilience, and academic outcomes. This is consistent with the hypothesis that students 


\begin{tabular}{|c|c|c|c|c|c|c|c|c|c|c|}
\hline Model & $R$ & $R^{2}$ & $R^{2}$ change & $F$ & $\mathrm{df}$ & $p$ & $\begin{array}{l}\text { Unstandardized } \\
B\end{array}$ & $\begin{array}{l}\text { Standardized } \\
\text { beta }\end{array}$ & $t$ & $p$ \\
\hline Block 1 & .17 & .03 & .03 & 1.37 & 1 & .25 & - & -- & - & -- \\
\hline H S GPA & -- & -- & -- & -- & -- & -- & -.68 & -.30 & -1.98 & .06 \\
\hline Block 2 & .17 & .03 & .00 & .66 & 2 & .96 & - & -- & -- & -- \\
\hline PAP & -- & -- & -- & -- & - & -- & .01 & .01 & .42 & .70 \\
\hline Block 3 & .38 & .14 & .11 & 2.33 & 3 & .02 & -- & - & -- & -- \\
\hline Life Events & - & - & - & - & -- & - & -.04 & -.37 & -2.35 & .02 \\
\hline
\end{tabular}

Note. Block 1 = High School (HS) GPA, block 2 = perceived academic preparedness (PAP), block 3 = stressful/traumatic life events (weighted).

from low socioeconomic status backgrounds may be more similar to than different from other students. Future work examining some of the constructs from this research in non-enrolled emerging adults from a low socioeconomic backgrounds could provide valuable information regarding this hypothesis.

Regression analyses indicated that a history of life events accounts for approximately $11 \%$ or $14 \%$ of the variance in grade-related outcomes, and for each additional life event reported, a student is $24 \%$ more likely to withdraw from a course. Furthermore, although many of the expected correlations did not reach significance, they had medium effect sizes, indicating a practical importance. Taken together, although much variability still needs to be explained, it appears that event history is related to academic outcomes. Future work should examine larger samples of students for longer periods to better understand this process.

In addition to primary findings, there were other noteworthy results from the present study. One is that self-reported resilience was associated with other self-reported resiliency factors, consistent with prior research (Smith et al., 2008). Given the complexities of resilience, and the ways in which it is measured, future work would benefit from continuing to determine relationships between self-report measures, while also moving toward the inclusion of behavioral measures. For example, prior research indicates presence of a mentor, particularly during emerging adulthood, may be a resiliency factor. Therefore, future work could track number of meetings with advisors to provide evidence of how behavioral information may be similar/dissimilar to self-report data.

Table 6
The second of these is that all of the academic outcome variables significantly correlated with one another. Although not a primary aim of the study, three potential academic functioning outcome variables were included to enable a broadened definition of academic functioning. Past research has utilized primarily GPA, limiting the conclusions that can be drawn due to the many correlates of GPA (Richardson, Abraham, \& Bond, 20I2). Although Fall course withdrawals and Fall D/F grades were related to GPA, course withdrawals in particular showed differential relationships with other variables in the study. Therefore, this variable may provide information that is unique from gradebased outcomes. Future research should pursue this, to determine where these outcomes overlap, and where they provide distinct information from one another.

A final noteworthy result is the divergent relationships between the experience of an event (unweighted score) and the perception of an event's impact (weighted score). Specifically, while number of experienced events was significantly associated with depression and anxiety, as well as course withdrawals, perception of event did not significantly correlate with these three variables.

One interpretation of this discrepancy is the ambiguous wording of the required follow-up question. This question asked respondents to rate how much the event had affected life in the past year. Respondents, therefore, are left to decide for themselves whether this effect was positive or negative. There has been a growing literature addressing the concept of post-traumatic growth, or positive changes individuals may experience following a stressful and traumatic event. For example, some individuals report improved relationships or increased appreciation

Summary of Regression Analysis of GPA

\begin{tabular}{|c|c|c|c|c|c|c|c|c|c|c|}
\hline Model & $R$ & $R^{2}$ & $R^{2}$ change & $F$ & $\mathrm{df}$ & $p$ & $\begin{array}{l}\text { Unstandardized } \\
B\end{array}$ & $\begin{array}{l}\text { Standardized } \\
\text { beta }\end{array}$ & $t$ & $p$ \\
\hline Block 1 & .13 & .02 & .02 & .78 & 1 & .38 & - & - & -- & -- \\
\hline HS GPA & -- & -- & - & - & - & -- & .64 & .28 & 1.82 & .08 \\
\hline Block 2 & .13 & .02 & .00 & .38 & 2 & .98 & -- & -- & -- & -- \\
\hline PAP & -- & -- & -- & -- & -- & -- & -.00 & -.07 & -.50 & .62 \\
\hline Block 3 & .40 & .16 & .14 & 2.64 & 3 & .01 & -- & -- & -- & -- \\
\hline Life Events & -- & -- & -- & -- & -- & -- & -.04 & .41 & 2.67 & .01 \\
\hline
\end{tabular}

Note. Block 1 = High School (HS) GPA, block 2 = perceived academic preparedness (PAP), block $3=$ stressful/traumatic life events (weighted) 
for life (Tedeschi, \& Calhoun, 1996). It is not possible to determine if students in the present study interpreted the question this way; however, future work could consider this interpretation.

Although this is possible, the results were consistent with prior work on academic outcomes - more stress and trauma leads to worse outcomes. Therefore, another hypothesis is that the results of this study represent a legitimate difference between the sheer number of events experienced versus the perception of how these events are affecting oneself (cognitive appraisal) Indeed, some past research has reported that the emotional response and perception of the event are what actually relate to outcomes, such as PTSD, not the event itself (Boals, \& Schuettler, 2009). Given the lack of available literature in this area, future work is needed to clarify how the experience of events may be moderated by emotional response and cognitive appraisal to affect outcomes. Put differently, are these non-clinical processes naturally at work in students' lives that can have the same beneficial impact as clinical intervention? If so, what might these be and how can we, as educators, facilitate this process?

\section{Limitations}

There are a number of limitations to the present study. Primary among these is the reliance on self-report measures. Self-report measures, though commonly used in research, rely on the individual completing them to be forthcoming, as well as have the insight to complete the measures accurately. Furthermore, the PTSD screener was not completed by all students, seemingly due to not understanding the instructions. Given that this research was interested in trauma, not being able to examine the relationship between the variables of interest and PTSD symptoms is problematic. Future work would benefit from using a different measure or perhaps a clinical interview to better understand PTSD and other mental health symptoms.

Another limitation of the present study is that there was only one time point available for the academic outcome variables. Past work indicates that the first year, and the first semester of the first year, may be particularly important to the academic course of students (Boyraz et al., 20I5). However, having only the first semester data made it difficult to examine some potentially important outcomes, as the ranges of some of the outcome variables (course withdrawals and number of D/F grades) were relatively constricted. Furthermore, students may have been less likely to disclose during this period of adjustment and transition, or may have felt they needed to alter their behavior to make a favorable impression.Attempts were made to mitigate the effects of these limitations, such as by discussing confidentiality with the students prior to study participation. Future work will benefit from inclusion of further time points while students are in college. Despite these factors, a significant correlation was found between total number of life events reported and course withdrawals for the Fall semester, supporting the hypothesis of the present study and past research.

A final limitation was the lack of a direct comparison sample. With the exception of data from the BCSSE, there was no way to determine whether the group of students in this study was similar to or different from the broader sample of students at the university. Results indicate that on the BCSSE, the present sample was mostly similar to the student body. Additionally, although it was difficult to compare this sample to those in other research due to the use of medians in the present study and the use of means in other research, it seems there may be differences in this sample (e.g., less anxiety/depression in present sample). However, without a direct comparison, it is difficult to know whether or not this is a function of the unique sample in this study.

\section{CONCLUSION}

Our study is consistent with those reporting that trauma and stress prior to college admission contribute to a range of poor academic performances while in college. On a more positive note, and perhaps of most importance, is the absence of evidence that students from extremely poor economic backgrounds experience any more trauma and stress than their more advantaged counterparts. Further, the students in our cohort reported only minimal frequency and intensity of mental health difficulties. This may reflect unacknowledged admission biases that select for the more resilient and mentally healthy students (aside from high school academic performance), which only future research can address. The immediate practical implication of our results suggest that we not presume that our economically poorer students, once admitted, are more poorly prepared for college. Finally, there may be a gender difference, with female students reporting trauma and stress but male students reporting more intensive reactions, that require attention as we refine our efforts to address the mental health needs of our students.

\section{REFERENCES}

American Psychiatric Association. (2013). Diagnostic and statistical manual of mental disorders: DSM-5. Washington, D.C:Author.

Ailes II, L.P.,Alvarado, N.,Amundson, S., Bruchey, J., \& Wheeler, C.J. (20I7). Expectations versus reality: First-year students' transition into residential living. Journal of the Student Personnel Association at Indiana University, 34-48.

Anders, S.L., Frazier, P.A., \& Shallcross, S.L. (20I2). Prevalence and effects of life event exposure among undergraduate and community college students. Journal of Counseling Psychology, 59(3), 449-457. doi 10.1037/a0027753

Bachrach, R.L., \& Read, J.P. (20I2). The role of posttraumatic stress and problem alcohol involvement in university academic performance. Journal of Clinical Psychology, 68(7), 843859. doi 10.1002/jclp.21874

Baker, R.W., McNeil, O.V., Siryk, B. ( 1985). Expectation and reality in freshman adjustment to college. Journal of Counseling Psychology, 32(I), 94-I03.

Baron, R.M., \& Kenny, D.A. ( 1 986). The moderator-mediator variable distinction in social psychological research: Conceptual, strategic, and statistical considerations. Journal of Personality and Social Psychology, 5 I, I I73-I I 82.

Beck, A.T., \& Steer, R.A. (1990). Manual for the Beck anxiety inventory. San Antonio, TX: Psychological Corporation.

Beginning College Survey of Student Engagement. (2010). Beginning College Survey of Student Engagement. Retrieved from http://bcsse.iub.edu/

Beiter, R., Nash, R., McCrady, M., Rhoades, D., Linscomb, M., Clarahan, M., \& Sammut, S. (20I5). The prevalence and correlates of depression, anxiety, and stress in a sample of college students. Journal of Affective Disorder, I73, 90-96. doi 10.1016/j. jad.2014.10.054

Boals, A., Schuettler, D. (2009). PTSD symptoms in response to traumatic and non-traumatic events:The role of respondent perception and A2 criterion. Journal of Anxiety Disorder, 23, 
458-462. doi 10.1016/j.janxdis.2008.09.003

Bonanno, G.A., \& Mancini, A.D. (2008). The human capacity to thrive in the face of potential trauma. Pediatrics, I 2 I (2), 369375. doi 10.1542/peds.2007-1648

Boyraz, G. Granda, R., Baker, C.N., Tidwell, L.L., \& Waits, J.B. (20I5). Posttraumatic stress, effort regulation, and academic outcomes among college students:A longitudinal study. Journal of Counseling Psychology. doi 10.1037/cou0000I02

Boyraz, G., Horne, S.G., Owens, A.C., \& Armstrong, A.P. (20I3). Academic achievement and college persistence of African-American students with trauma exposure. Journal of Counseling Psychology, 60(4), 582-592. doi 10.1037/a0033672

Carmody, D.P. (2005). Psychometric characteristics of the beck depression inventory in college students of diverse ethnicity. International Journal of Psychiatry in Clinical Practice, 9(1), 2228. doi 10.1080/1365/500510014800

Doom, J.R., \& Haeffel, G.J. (20I3). Teasing apart the effects of cognition and depression on health. American Journal of Health Behavior, 37, 610-619.

Duncan, R.D. (2000). Childhood maltreatment and college dropout rates: Implications for child abuse researchers. Journal of Interpersonal Violence, I 5(9), 987-995.

Duncan, G.J., Brooks-Gunn, J., Yeung, W.J., \& Smith, J.R. (1998). How much does childhood poverty affect the life chances of children? American Sociological Review, 63(3), 406-423.

Eisenberg, D., Golberstein, E., \& Gollust, S.E. (2007). Help-seeking and access to mental health care in a university student population. Medical Care, 45(7), 594-60I. doi I0.I097.MLR0b0I3e31803bb4cl

Elhai, J. D., Miller, M. E., Ford, J. D., Biehn, T. L., Palmieri, P. A., \& Frueh, B. C. (20I2). Posttraumatic stress disorder in DSM-5: Estimates of prevalence and symptom structure in a nonclinical sample of college students. Journal of Anxiety Disorders, 26(I), 58-64. doi: I0.1016/j.janxdis.201 I.08.013

Freeman, D., \& Fowler, D. (2009). Routes to psychotic symptoms: Trauma, anxiety, and psychosis-like experiences. Psychiatry Research, 169, 107-I I2. doi 10.1016/j.psychres.2008.07/009

Goldrick-Rab, S., Kelchen, R., Harris, D. N., \& Benson, J. (20I6). Reducing income inequality in educational attainment: Experimental evidence on the impact of financial aid on college completion. American Journal of Sociology, I2 I (6), I762-1817.

Hardaway, C.R., Larkby, C.A., \& Cornelius, M.D. (20I4). Socioemotional adjustment as a mediator of the association between exposure to community violence and academic performance in low-income adolescents. Psychology of Violence, 4(3), 28I-293. doi 10.1037/a0036375

Harackiewicz, J.M., Barron, K.E., Tauer, J.M., \& Elliot, A.J. (2002). Predicting success in college:A longitudinal study of achievement goals and ability measures as predictors of interest and performance from freshman year through graduation. Journal of Educational Psychology, 94(3), 562-575. doi 10.1037//0022-0663.94.3.562

Hunt, J., \& Eisenberg, D. (2010). Mental health problems and help-seeking behavior among college students. Journal of Adolescent Health, 3-10. doi 10.1016/j.jadohealth.2009.08.008

Infurna, F.J., \& Luthar, S.S. (2016). Resilience to major life stressors is not as common as thought. Perspectives on Psychological Science, I I (2), I75-194. doi I0. I I77/I74569 I6I562 I 27I

Jansen, K.L., Motley, R., \& Hovey, J. (2010).Anxiety, depression and students' religiosity. Mental Health, Religion, and Culture, I3(3),
267-27I. doi 10.1080/I3674670903352837

Kessler, R.C., Foster, C.L., Saunders, W.B., \& Stang, P.E. (1995). Social consequences of psychiatric disorders, I: Educational attainment. American Journal of Psychiatry, 152, I026- 1032.

Kraemer, H.C., Kiernan, M., Essex, M., \& Kupfer, D.J. (2008). How and why criteria defining moderators and mediators differ between the Baron \& Kenny and MacArthur approaches. Health Psychology, 27(2), I0I-I08. doi 10.1037?0278-6I33.27.2(Suppl.).SI0I

Krieg, D. (2013). High expectations for higher education? Perceptions of college and experiences of stress prior to and through the college career. College Student Journal, 47(4), 635-643.

Martin, J.A., \& Elmer, E. ( 1992). Battered children grown up:A follow-up study of individuals severely maltreated as children. Child Abuse and Neglect, 16, 75-87.

Masten, A.S. (200I). Ordinary magic: Resilience processes in development. American Psychologist, 56(3), 227-238. doi 10.1037/0003-066X.56.3.227

Masten, A.S., Burt, K.B., Roisman, G.I., Obradovic, J., Long, J.D., \& Tellegen,A. (2004). Resources and resilience in the transition to adulthood: Continuity and change. Development and Psychopathology, I6, I07I-I094. doi I0. I0I7/S0954579404040 I43

McHugo, G.J., Kammerer, N., Jackson, E.W., Markoff, L.S., Gatz, M., Larson, M.J., ... \& Hennigan, K. (2005).Women, co-occurring disorders, and violence study: Evaluation design and study population. Journal of Substance Abuse Treatment, 28(2), 91 107.

Mojtabai, R., Stuart, E. A., Hwang, I., Eaton, W.W., Sampson, N., \& Kessler, R. C. (20I5). Long-term effects of mental disorders on educational attainment in the National Comorbidity Survey ten-year follow-up. Social Psychiatry and Psychiatric Epidemiology, 50(10), I577-I59I.

Perez, C.M., \& Widom, C.S. (1994). Childhood victimization and long-term intellectual and academic outcomes. Child Abuse and Neglect, I8(8), 617-633.

Prins, A., Ouimette, P., Kimerlin, R., Cameron, R.P., Hugelshofer, D.S., Shaw-Hegwer, A. T., ...Sheikh, J.I. (2003). The primary care PTSD screen (PC-PTSD): Development and operating characteristics. Primary Care Psychiatry, 9(I), 9-I4.

Prins, A., Bovin, M.J., Smolenski, D.J., Marx, B.P., Kimerling, R., Jenkins-Guarnieri, M.A.,...Tiet, Q.Q. (20I5). The primary care PTSD screen for DSM-5 (PC-PTSD-5): Development and evaluation within a veteran primary care sample. Journal of General Internal Medicine, 3 I(10), I206-121I. doi 10.1007/ sl |606-0|6-3703-5

Read, J.P., Ouimette, P., White, J., Colder, C., \& Farrow, S. (20I I). Rates of DSM-IV-TR trauma exposure and posttraumatic stress disorder among newly matriculated college students. Psychological Trauma:Theory, Research, Practice, and Policy, 3(2), |48-I56. doi 10.1037/a002 I 260

Richardson, M., Abraham, C., \& Bond, R. (20I2). Psychological correlates of university students' academic performance: A systematic review and meta-analysis. Psychological Bulletin, I 38(2), 353-387. doi 10.1037/a0026838

Smith, B.W., Dalen, J., Wiggens, K., Tooley, E., Christopher, P., \& Bernard, J. (2008). The brief resilience scale: Assessing the ability to bounce back. International Journal of Behavioral Medicine, 15(3), 194-200.

Steer, R.A., \& Clark, D.A. (1997). Psychometric characteristics of 
the beck depression inventory-II with college students. Measurement and Evaluation in Counseling and Development, 30(3).

Tedeschi, R.G., \& Calhoun, L.G. (1996). The posttraumatic growth inventory: Measuring the positive legacy of trauma. Journal of Traumatic Stress, 9(3).

Ungerer, O., Deter, H.C., Fikentscher, E., \& Konzag, T.A. (20I0). Improved diagnostics of trauma-related disease through the application of the Life-Stressor Checklist. Psychotherapie, Psychosomatik, Medizinische Psychologie, 60(I I), 434-44I. doi 10.1055/s-0030-1247497
University of Louisville. (2016). BCSSE Institutional Report. Watkins, D. (1978).A note on student satisfaction with university: A case study. Education Research and Perspectives, 5, 45-53.

Wolfe, J., Kimerling, R., Brown, P.J., Chrestman, K.R., \& Levin, K. (1996). Psychometric review of the life stressor checklist-revised. Measurement of stress, trauma, and adaptation, I98-20I. World Health Organization. (20l4). Social determinants of mental health. World Health Organization. 